\title{
Decentralized Control of Large-Scale Networks as a Game with Local Interactions: Cross-layer TCP/IP Optimization
}

\author{
Vladimir Marbukh \\ National Institute of Standards and Technology \\ 100 Bureau Drive, Stop 8920, \\ Gaithersburg, MD 20899-8920, USA \\ Tel: 13019752235 \\ E-mail: marbukh@nist.gov
}

\author{
Stephan Klink \\ National Institute of Standards and Technology \\ 100 Bureau Drive \\ Gaithersburg, MD 20899-8920, USA \\ Tel: 13019752235 \\ E-mail: klink@gmx.net
}

\begin{abstract}
Developing optimized distributed protocols for large-scale networks is a challenging problem due to scalability and stability concerns. Scalability concerns can be naturally addressed by interpreting distributed protocols as a non-cooperative game of local protocol components attempting to maximize their individual utilities. One of the difficulties in implementing this approach is developing adaptive algorithms capable of learning of the expected utilities and adjusting the corresponding control actions for the purpose of approaching the solution to the corresponding game, and thus optimization of the global system performance. It is known that the best response by each component to its expected utility may result in unstable behavior and deterioration of the overall performance. On an example of cross-layer optimization of a TCP/IP network, this paper discusses the possibility of avoiding these undesirable effects by allowing the control actions occasionally deviate from their best response values. Using simulations, the paper suggests that (a) sufficient level of randomness in route selection improves the network performance by eliminating the route flapping instability, (b) the optimal level of randomness keeps the network within the stability region in close proximity to the border of this region, and (c) it may be possible to optimize the network performance by adjusting the level of randomness.
\end{abstract}

\section{Categories and Subject Descriptors}

C.4 [Performance of Systems]: - modeling techniques, performance attributes, reliability, availability, and serviceability.

\section{General Terms}

Algorithms, Management, Performance, Design, Reliability, Theory.

\section{Keywords}

Distributed protocols, optimization, learning algorithms, game theory, TCP/IP, OSPF, randomized routing, routing stability.

Permission to make digital or hard copies of all or part of this work for personal or classroom use is granted without fee provided that copies are not made or distributed for profit or commercial advantage and that copies bear this notice and the full citation on the first page. To copy otherwise, or republish, to post on servers or to redistribute to lists, requires prior specific permission and/or a fee.

Valuetools'07, October 23-25, 2007, Nantes, France.

Copyright 2007 ICST 978-963-9799-00-4

\section{INTRODUCTION}

Recent technological advances made implementation of complicated adaptive network management algorithms technologically feasible [1]. Adaptive network management algorithms extract essential information from real-time or near real-time measurements and produce the appropriate responses. Developing optimized distributed protocols for large-scale, selfmanaged systems is a challenging problem due to scalability and stability concerns. Scalability concern can be naturally addressed by interpreting distributed protocols as a non-cooperative game where each component is modeled as a player attempting to maximize its own utility [2]. Success of this game theoretic approach, however, requires solving two major problems. The first problem is identifying of the "optimal" individual utilities through resource pricing so that (hopefully unique) Nash equilibrium in this game optimizes the overall network performance. Since typically such "optimal” individual utilities are themselves solution to some global optimization problem, the second problem is developing adaptive algorithms capable of learning of the expected utilities and adjusting the corresponding control actions for the purpose of approaching the solution to the corresponding game, and thus optimization of the global system performance. Distributed, adaptive algorithms attempt to approach the global optimum by learning evolution, when each player makes its decisions based on the history of its own decisions and responses by other players [2]. Typically, if this evolutionary process converges, the corresponding steady state also represents the Nash solution to the corresponding game and thus optimizes the overall steady-state performance. The convergence, however, is not guaranteed even in a rather exception case of a pure Nash equilibrium in the corresponding game, let alone a common case of a mixed Nash equilibrium.

A classical networking example of such instability is route flapping in a situation of equal cost multipath [3]. It is known [4] that under certain conditions the necessary and sufficient condition for the routing algorithm to achieve optimal load balancing is to select minimum cost route, where the link costs are increasing and convex functions of the link loads. However, adaptive version of this routing, which estimates the average link loads, and then routes the entire flow on the minimum cost path, increases load on the minimum cost route until flow admission strategy takes over or at least two feasible routes have approximately the same cost. After that the routing decisions become very sensitive to the route costs causing route flapping instability, which significantly increases the amount of signaling traffic and may cause deterioration in the network performance [5]. Conventional approach to ensuring convergence to the 
optimum is switching only a small (infinitesimal) portion of traffic on the minimum cost route [6]. Except for MPLS, currently existing routing algorithms, such as Open Shortest Path First (OSPF) and its Traffic Engineering (TE) extensions require flow level granularity in load balancing, i.e., all packets of the same flow are to be routed on the same minimum cost path to avoid out of order arrivals [3].

Practical protocol implementations usually offer some phenomenological mechanisms for alleviating instability problems. For example, OSPF routing protocol assumes uniform traffic split among multiple equal minimum cost routes in order to achieve better load balancing and allows minimum period of 30 seconds for the link cost updates [3]. New technological advances allow for more flexibility in tuning the protocols to specific conditions. Taking advantage of these emerging capabilities requires better understanding of various trade-offs involved in protocol design, including stability/performance trade-off. Possibility of stabilization of a minimum cost routing by adding the load independent component to link costs has been discussed in [5], [7]-[8]. The main conclusion is that stability generally requires the relative weight on the dynamic (load sensitive) component of the link cost to be sufficiently small. Increase of the dynamic component improves the network performance until the performance peaks and then deteriorates. Deterioration in the network performance occurs close to the point of loosing stability.

Recently, IP community expressed interest in relaxing OSPF requirement to split load equally among feasible routes of minimum cost in a situation of equal cost multi-path [9]-[10]. Paper [9] has demonstrated that any (including optimal) routing can be viewed as a minimum cost routing based on some link costs assuming, however, the ability to split traffic arbitrarily among multiple feasible minimum cost routes. Paper [9] has argued that technologically such ability may be achieved in IP network running OSPF or IS-IS protocols by manipulating the set of next hops for route prefixes. Due to inherent uncertainties in the external demands and network conditions, e.g., due to link failures, it is natural to attempt to determine the set of optimal, i.e., minimum cost, routes and the optimal load split among these routes adaptively, based on the on-line measurements. This paper is a preliminary attempt to identify approach to this problem in a context of cross-layer optimization of a TCP/IP network. The paper proposes a scheme allowing flows to be routed on "almost" minimum cost paths. The load split among "almost" minimum cost routes is optimized by sending more flows with the same source-destination on the paths with lower estimated costs.

The proposed scheme allowing routing decisions occasionally deviate from their optimal (minimum estimated cost) route selection has natural interpretation as a learning or evolutionary algorithm. One may expect that adding "noise” to the system dynamics may stabilize the system in some neighborhood of the optimal state, trading steady-state performance under quasistationary conditions for robustness under unexpectedly changing conditions. The question we are most interested in this paper is stabilization and optimization of the network by such randomization. Note the difference between this view and view accepted in genetic algorithms, where algorithm mutation is basically a discovery mechanism.

The paper considers a TCP/IP network where TCP-AQM flow control mechanism is assumed to maximize the aggregate utility [5]. We assume that a single route is selected for each arriving flow, and that TCP-AQM instantaneously maximizes the aggregate utility. These assumptions reflect flow level granularity in load allocation and much faster TCP-AQM convergence then a flow arrival/departure process. Section 2 abstracts TCP operations as an aggregate utility maximization problem for a given set of flows. Section 3 characterizes the optimal flow level granularity routing and introduces a noncooperative game $G^{*}$ with a Nash equilibrium representing the optimal routing. Learning algorithm attempting to approach this equilibrium by the best responses to the expected utility represents the minimum cost routing and may exhibit route flapping instability causing sharp deterioration in the network performance. Section 4 describes a minimum average cost routing representing the stochastic fictitious play in the corresponding game $G^{*}$. Section 5 describes a model for the network dynamics with the minimum average cost routing and random number of flows in progress due to random flow arrivals/departures. Section 6 reports simulation results indicating that (a) sufficient level of randomness improves the network performance by eliminating the route flapping instability, (b) the optimal level of randomness keeps the network within the stability region in close proximity to the border of this region, and (7) it may be possible to adaptively optimize the level of randomness. Finally, conclusion summarizes the results and outlines direction for future research.

\section{MODEL}

A network is comprised of a set of nodes $n \in N$ connected by bi-directional links $l \in L$ with finite capacities $C_{l}$. Traffic with source-destination $S \in S$ can be routed over feasible acyclic paths $r \in R_{s}$. We assume that there are $M_{s}=\operatorname{dim} K_{s}$ users $k \in K_{s}$ generating demand with source-destination $S$. Each user $k \in K_{s}$ has utility function $u_{s}(x)$ of transmitting at a rate $x \geq 0$, where functions $u_{s}(x)$ are strictly increasing and continuous.

It is known [11], [5], that TCP-AQM results in the bandwidth allocation maximizing the aggregate utility. Note that for TCP utility functions $u_{s}(x)$ are typically strictly concave. Assuming that user $k \in K_{s}$ traffic can be arbitrarily split among feasible routes $r \in R_{s}$, the aggregate utility maximization problem is as follows:

$$
\max _{X, Y} \sum_{s} \sum_{k \in K_{s}} u_{s}\left(\sum_{r \in R_{s}} x_{k r}\right)
$$

subject to the capacity constraints.

$$
y_{l} \leq c_{l}
$$

and flow non-negativity constraints

$$
x_{k r} \geq 0
$$

where $X_{k r}$ is the user $k \in K_{s}$ rate on a feasible route $r \in R_{s}$, and 


$$
y_{l}=\sum_{s} \sum_{k \in K_{s}} \sum_{r \in R_{s}} x_{k r}
$$

is the total link $l$ load.

Sometimes it is more convenient (and also relevant [11]) to replace capacity constraints (2) with penalty function in the optimized criterion. Consider the following optimization problem

$$
\max _{X, Y} W
$$

subject to constraints (3)-(4), where the social welfare is

$$
W=\sum_{s} \sum_{k \in K_{s}} u_{s}\left(\sum_{r \in R_{s}} x_{k r}\right)-\sum_{l} f_{l}\left(y_{l}\right)
$$

and penalty functions $f_{l}$ are monotonously increasing and convex:

$$
\begin{gathered}
d_{l}(y) \stackrel{\operatorname{def}}{=} d f_{l}(y) / d y>0, \\
d_{l}^{\prime}(y) \stackrel{\text { def }}{=} d^{2} f_{l}(y) / d y^{2}>0,
\end{gathered}
$$

$\forall y \in[0, \infty)$.

In this paper we assume that the entire user $k \in K_{s}$ flow

$$
x_{k} \stackrel{\text { def }}{=} \sum_{r \in R_{s}} x_{k r}
$$

is routed on a single path $r_{s k}$. It is straightforward to modify optimization problems (1)-(4) and (5)-(6) to account for this requirement by adding the corresponding integer constraints

$$
x_{k r}=\left\{\begin{array}{cll}
x_{k} & \text { if } & r=r_{s k} \\
0 & \text { if } & r \neq r_{s k}
\end{array}\right.
$$

Further in the paper we always assume that numbers of users with the same source-destinations are large:

$$
M_{s} \gg 1, \forall s \in S
$$

In this practically important case solution to the corresponding discrete optimization problem can be approximated based on the solution to the following continuous optimization problem:

$$
\max _{Z}\left\{\sum_{s} M_{s} u_{s}\left(\sum_{r \in R_{s}} z_{s r}\right)-\sum_{l} f_{l}\left(\sum_{s} M_{s} \sum_{r: l \in r \subseteq R_{s}} z_{s r}\right)\right\}
$$

subject to constraints $Z_{s r} \geq 0$. If solution to optimization problem (12) is known: $Z=\left(Z_{s r}\right)$, the optimal flow-level granularity routing algorithm allocates

$$
M_{s r} \approx \alpha_{s r} M_{s}
$$

users $S \in S$ on a route $r \in R_{s}$, where

$$
\alpha_{s r}=z_{s r} / \sum_{r^{\prime} \in R_{s}} z_{s r^{\prime}}
$$

In a case when user utilities $u_{s}(x)$ are concave, optimization problems (1)-(4) and (3)-(6) are convex and thus they have unique optimal solution $X^{*}$ and no other locally optimal solution exists.

\section{MINIMUM COST ROUTING}

A necessary condition for a vector $Z=\left(Z_{s r}\right)$ to be a solution to optimization problem (12) can be characterized in terms of the link costs (7) as follows [5]-[6]. A set of path flows is optimal if the flows are positive on feasible paths of minimum cost, where the cost of a path is a sum of the costs of the path's links:

$$
d_{r}=\sum_{l \in r} d_{l}
$$

and the link costs (7) are calculated at the point $Z=\left(Z_{s r}\right)$.

These necessary conditions are also sufficient if utilities $u_{s}(x)$ are concave and thus optimization problem (12) is convex. The characterization of the optimal load allocation in terms of the minimum cost routing implies that at the optimum the paths along which the load with the same source-destination $S \in S$ is split must have the same cost, which is the minimum cost over all feasible paths with the same source-destination $S$ (a situation of equal cost multi-path).

Given route costs $d_{r}$, consider individual optimization problem for a user $k \in K_{s}$

$$
\max _{r \in R_{s}} \max _{x \geq 0} \tilde{U}_{s}(r, x ; d)
$$

where the individual net utility is

$$
\tilde{U}_{s}(r, x ; d)=u_{s}(x)-x d_{r}
$$

Optimization problem (16)-(17) can be rewritten as follows:

$$
\max _{x \geq 0}\left\{u_{s}(x)-x \min _{r \in R_{s}} d_{r}\right\}
$$

with internal optimization having a form of a minimum cost routing:

$$
\min _{r \in R_{s}} d_{r}
$$

In absence of the equal cost multi-path situation solving individual optimization problem (16)-(17) by each user $k \in K_{s}, \quad s \in S$ independently from each other results in the optimal load allocation if the link cost $d_{l}$ are "right”. This simple observation suggests possibility of a distributed algorithm for optimization of the overall network performance. However, achieving this goal requires first, identifying the "right" link costs, an, second, dealing with equal cost multi-path situation when minimum cost route selection (19) is indifferent between two or more minimum cost routes.

Solving these problems requires accounting for the "interactions" among different users (flows) due to dependency of the actual route costs in each user individual net utility (17) not only on the routing and flow control decisions made by this user, but also on the routing and flow control decisions made by other users. Accounting for these interdependencies naturally leads to the following game theoretic interpretation of the optimal load allocation problem. Consider a non-cooperative game $G$ of all users $k \in K_{s}, s \in S$. User $k$ attempts to select its route $r_{k} \in R_{s}$ and rate $x_{k} \in[0, \infty)$ in order to maximize its utility

$$
U_{s}\left(r_{k}, x_{k} ; r_{-k}, x_{-k}\right)=u_{s}\left(x_{k}\right)-x_{k} d_{r_{k}}\left(r_{-k}, x_{-k}\right)
$$


where vector $r_{-k}$ includes routes and vector $X_{-k}$ includes rates for all users except user $k$. Note that expression (20) assumes (11). Solution to the user optimization problem

$$
\max _{r_{k} \in R_{s}} \max _{x_{k} \geq 0} U_{s}\left(r_{k}, x_{k} ; r_{-k}, x_{-k}\right)
$$

depends on the decisions by other users. Note that this game theoretic formulation assumes that instantaneous information on the link costs is available to the users and users can instantaneously react to this information by adjusting their routes and transmission rates. In practical applications, the frequency of link cost updates is upper bounded, e.g., for OSPF routing protocol the shortest period between link costs updates is 30 seconds [3]. Section 5 accounts for finite frequency of link cost updates in the dynamic model of route selection.

Due to much faster TCP-AQM convergence than frequency of routing updates we assume that given routes for all users, rates $x_{k}=x_{k}^{*}$ maximize the criterion (6). This can be justified when user utility functions $u_{s}(x)$ are typically strictly concave and thus game $G$ in rates $\left(x_{k}\right)$ for given routes $\left(r_{k}\right)$ have unique pure equilibrium $\left(x_{k}^{*}\right)$, where $x_{k}^{*}=z_{s}^{*}, k \in K_{s}$ and $\left(z_{s}^{*}\right)$ is the unique solution to the following optimization problem

$$
\max _{x_{s} \geq 0}\left\{\sum_{s} M_{s} u_{s}\left(z_{s}\right)-\sum_{l} f_{l}\left(\sum_{s} M_{s} z_{s} \sum_{r: l \in r \subseteq R_{s}} \alpha_{s r}\right)\right\}
$$

for given portions of flows routed on different feasible paths

$$
\alpha_{s r}=\sum_{k \in K_{s}} \delta\left(r_{k}, r\right) / \sum_{r \in R_{s}} \sum_{k \in K_{s}} \delta\left(r_{k}, r\right)
$$

where $\delta(i, j)=1$ if $i=j$ and $\delta(i, j)=0$ otherwise. Under this assumption routing and flow control game $G$ simplifies into routing game $G^{*}$ where each user $k \in K_{s}$ selects its route $r_{k}$ in an attempt to minimize the route cost:

$$
\min _{r_{k} \in R_{s}} d_{r}^{*}\left(r_{-k}\right)
$$

where the route costs are calculated assuming that user rates have already reached the optimum $x_{-k}=x_{k}^{*}$ :

$$
d_{r}^{*}\left(r_{-k}\right)=d_{r}\left(r_{-k}, x_{-k}^{*}\right)
$$

Importance of the game $G^{*}$ follows from the following two observations: (a) minimum cost routing (19) can be viewed as the best user response in the game $G^{*}$, where $d_{r}=\tilde{d}_{r}$ are the observed route costs, (b) there is a close relation between Nash equilibrium strategies in the game $G^{*}$ and solution to optimization problem (12). It can be shown that each local maximum in (12) corresponds to Nash equilibrium in the game $G^{*}$, where user $k \in K_{s}$ selects strategy $r_{k} \in R_{s}$ with probability $\alpha_{s r}$, given by (14). A local solution to (12), which sends entire traffic with the same source-destination $S$ on a single feasible route $r_{s}^{*} \in R_{s}$, corresponds to pure equilibrium strategy $r_{k}=r_{s}^{*}$ for users $k \in K_{s}$ in the game $G^{*}$. Solution to (12), which splits traffic with the same source-destination $S$ among feasible routes $r \in R_{s}$, corresponds to mixed strategy for users $k \in K_{s}$ in the game $G^{*}$ : select route $r \in R_{s}$ with probability $\alpha_{s r}$, given by (14).

\section{MINIMUM AVERAGE COST ROUTING}

The following minimum average cost routing reduces sensitivity of the routing decisions to the instantaneous link utilization by randomizing routing decisions. Given the observed route costs $d_{r}=\tilde{d}_{r}$, each user $S$ selects a route $r \in R_{s}$ with probability $\beta_{s r}$, where distribution $\beta_{s}=\left(\beta_{s r}, r \in R_{s}\right)$ minimizes the average route cost

$$
D_{s}=\min _{\beta_{s}} \sum_{r \in R_{k}} \beta_{s r} d_{r}
$$

subject to conditions $\sum \beta_{s r}=1, \beta_{s r} \geq 0, r \in R_{s}$ and the following condition

$$
-\sum_{r \in R_{k}} \beta_{s r} \log \beta_{s r} \geq h_{s}
$$

requiring a certain level of randomness in the route selection measured by the entropy of this selection $h_{s}$.

It is known [12] that solution to (26)-(27) is as follows

$$
\beta_{s r}=\left\{\begin{array}{cll}
e^{A_{s}-\gamma d_{r}} & \text { if } & r \in R_{s} \\
0 & \text { if } & r \notin R_{s}
\end{array}\right.
$$

where

$$
A_{s}=-\log \sum_{r \in R_{s}} e^{-\gamma d_{r}}
$$

and $\gamma=\gamma_{s}$ is the unique solution to equation

$$
-A_{s}+\not D_{s}=h_{s}
$$

The average route cost (24) can be expressed as follows

$$
D_{s}=d A_{s}(\gamma) / d \gamma
$$

It is easy to verify that a case $h_{s}=0$ or, equivalently, $\gamma_{s}=\infty$, corresponds to the minimum cost routing selection, and that a case $h_{s}=\log \left(\operatorname{dim} R_{s}\right)$ or, equivalently $\gamma_{s}=0$, corresponds to the equal flow split among $\operatorname{dim} R_{s}$ feasible routes $r \in R_{s}$. Figure 1 sketches the probability $\beta_{1}$ of route $r_{1}$ selection as a function of the difference in the route costs $d_{2}-d_{1}$ in a case of two feasible routes: $R_{s}=\left\{d_{1}, d_{2}\right\}$. Note, that procedure (28)-(30) can be easily generalized to include routing only over a subset of "almost" minimum cost 
feasible routes $r \in R_{s}^{*} \subseteq R_{s}$, e.g., routes having "sufficiently" low cost.

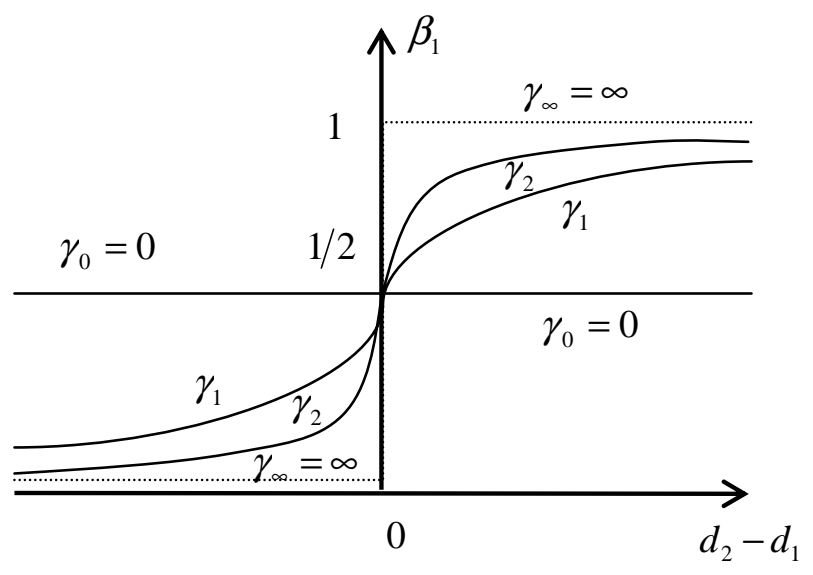

Fig. 1. Probability of route $r_{1}$ selection

$$
\left(0=\gamma_{0}<\gamma_{1}<\gamma_{2}<\gamma_{\infty}=\infty\right) \text {. }
$$

A minimum average cost route selection with positive entropy $h>0$, or equivalently finite parameter $\gamma<\infty$, reduces the overall system performance measured by the criterion (6), comparatively to a case of the minimum cost routing with $h=0$, or equivalently $\gamma=\infty$, assuming that this minimum cost routing is stable. For small entropy $h>0$, or equivalently large parameter $\gamma<\infty$, this loss in performance can be estimated as follows:

$$
W^{o p t}-W \approx \sum_{s} a_{s}\left(D_{s}-D_{s}^{\min }\right)
$$

where the average and the minimum route costs for a user $k \in K_{s}$ are respectively $D_{s}$ and

$$
D_{s}^{\min }=\min _{r \in R_{s}} d_{s r}
$$

and $a_{s}>0$ are some constants. Expression (32) suggests minimum cost route selection (19), for which $D_{s}=D_{s}^{\min }$.

However, minimum cost routing may result in unstable behavior, which typically leads to deterioration on the network performance and undesirable transient effects. Stability requirements impose low limit on the average cost of a selected route (see Section 6 of the paper). Figure 2 sketches selection of the optimal operating point $\left(D_{s}\right)$ subject to the stability constraints in a case of two source-destinations $S=\{1,2\}$. The shape of the stability region can be obtained by stability analysis of the presented in the next section network dynamic equations. In this paper we only conjecture that this stability region is convex in the average route costs.

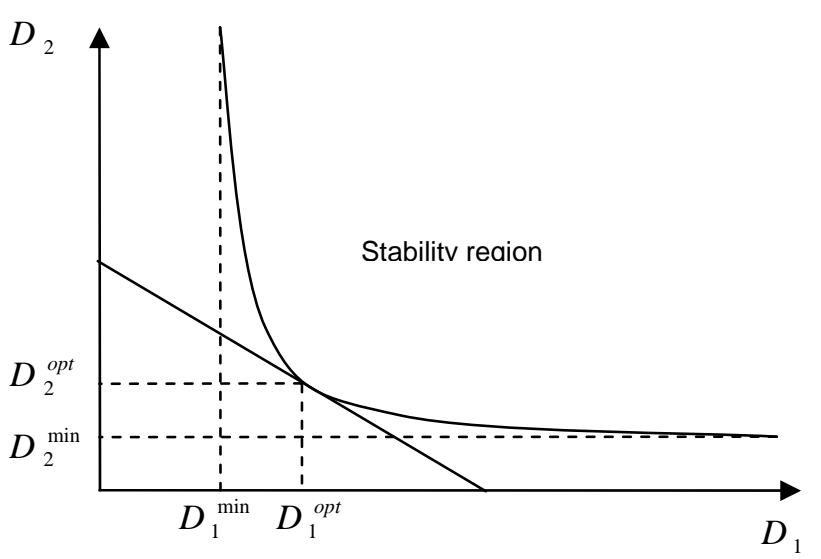

Fig. 2. Optimal operating point

\section{DYNAMICS AND LEARNING}

Consider the following model of TCP/IP operation. Link costs are updated and routing decisions are made at moments $t_{i}=i \tau, i=0,1,2, \ldots$. Flows arriving during time interval $t \in\left[t_{i}, t_{i+1}\right)$ are routed based on the link costs at the moment $t=t_{i}$, while "fast" TCP-AQM flow control keeps the transmission rates of all flows at the optimal levels all time. Thus, the number of flows $k \in K_{s}$ routed on a path $r \in R_{s}$ at step $i+1$ is

$$
M_{s r}(i+1)=\left\{\begin{array}{cll}
M_{s}(i) e^{A_{s}(i)-\gamma d_{r}(i)} & \text { if } & r \in R_{s} \\
0 & \text { if } & r \notin R_{s}
\end{array}\right.
$$

where the total number of flows $k \in K_{s}$ in the system at step $i$ is $M_{s}(i)$, constant

$$
A_{s}(i)=-\log \sum_{r \in R_{s}} e^{-\gamma d_{r}(i)},
$$

$\gamma=\gamma_{s}(i)$ is the unique solution to equation

$$
-A_{s}(\gamma)+\gamma \sum_{r \in R_{s}} \beta_{s r} d_{r}=h_{s}(i),
$$

route $r$ cost is

$$
d_{r}(i)=\sum_{l \in r} d_{l}(i)
$$

link $l$ cost is

$$
d_{l}(i)=\left[d f_{l}(y) / d y\right]_{y=y_{l}(i)},
$$

link $l$ load at step $i$ is

$$
y_{l}(i)=\sum_{s} \sum_{r \in R_{s}} M_{s r}(i) x_{r}(i)
$$

and the rate of a flow $k \in K_{s}$ is

$$
x_{r}(i)=\arg \max _{x \geq 0}\left\{u_{s}(x)-x d_{r}(i)\right\}
$$

Note that in a case of file transfer, with Poisson flow arrivals and exponentially distributed file sizes, vector 
$\left(M_{s r}(i): r \in R_{s}, s \in S\right)$ forms a Markov chain for $i=0,1,2, . . \quad$ A case of a fixed set of flows can be obtained by assuming the total number of flows $k \in K_{s}$ in the system is fixed $M_{s}(i)=M_{s}, s \in S$. In a case of a large numbers of flows in progress (11), zero order approximation of evolution of Markov chain $\left(M_{s r}(i): r \in R_{s}, S \in S\right)$ is described by a closed system of deterministic "averaged" equations for the vector of averages $\left(E\left[M_{s r}(i)\right]: r \in R_{s}, s \in S\right)$. In a case when optimization problem (12) has a solution, which does not split demand with the same source-destination among multiple feasible routes, this optimum is an equilibrium for the averaged system. As it has been shown in [5], even in this case, the equilibrium may be unstable for the minimum cost routing (34)(40) with $h_{s}(i) \equiv 0$, or equivalently, $\quad \gamma_{s}(i) \equiv \infty$, $s \in S, \quad i=0,1,2, \ldots$ This "route flapping instability" is typical in a general case when optimization problem (12) has a solution, which splits demand with the same source-destination among multiple feasible routes. The instability can be prevented by reducing sensitivity of the link costs to the link utilization. Paper [5] has considered such a reduction by adding a static component to the link costs in a case when optimization problem (12) has a solution, which does not split demand with the same source-destination among multiple feasible routes.

We propose reducing sensitivity of the link costs to the link utilization with minimum average cost routing (34)-(36) by controlling entropies $h_{s}(i)$, or equivalently, parameters $\gamma_{s}(i)$, for $s \in S, \quad i=0,1,2, .$. in a general case when optimization problem (12) has a solution, which splits demand with the same source-destination among multiple feasible routes. Game theoretic interpretation offers important insights into this problem. A minimum average cost routing (34)-(36) represents a smooth (logistic) fictitious play [2] in the game $G^{*}$ allowing one to leverage relevant results on the learning in games for distributed protocol optimization. For example, known results [2] obtained by application of stochastic approximation to learning algorithms suggest that randomized routing (34)-(36) with large $\gamma_{s} \rightarrow \infty$ and link costs averaged over a large number of link cost update cycles results in load allocation close to the optimal.

Note, that the game theoretic interpretation of the minimum average cost routing algorithm suggests other rationales for randomization of the routing decisions besides elimination instability, such as unavoidable uncertainties in the operating environment, such as link failures, and "protection" against malicious attempts to manipulate the link cost values by an adversary/adversaries [2], [13]-[14]. Also note that the minimum average cost routing can be interpreted as an evolutionary algorithm, where attempt to minimize the route cost by each user $k \in K_{s}$ represents selection while randomization of the routing decisions represents mutations. The optimal operating point on Fig. 2 represents the optimal level of mutations.

\section{SIMULATION RESULTS}

This section presents simulation results indicating benefits of randomization of the routing decisions and possibility of adaptive optimization by adjusting the level of the randomness based on observed network stability/instability. We uses the latest version (v.2.01) of the GLASS simulation tool [15]. We extended the tool so that it provided periodical link cost updates and added a module that calculated the link costs for each feasible route and made the minimum average cost routing decisions. In order to investigate the possibility of adaptation we also implemented a module capable of observing history of route costs for the last several link cost update periods. Note that the simulation has reflected only the "IP part" of the TCP/IP cross-layer optimization. GLASS simulation has assumed that each arriving flow occupied a fixed bandwidth. This assumption corresponds to a particular case of TCP/IP for rigid streaming applications with utility function closely approximated by a step-wise function.

Figure 3 demonstrates existence of the optimal level of the randomness, represented by parameter $\gamma^{\text {opt }} \approx 0.6$ for a fully connected network with 12 nodes and a Poisson flow arrivals.

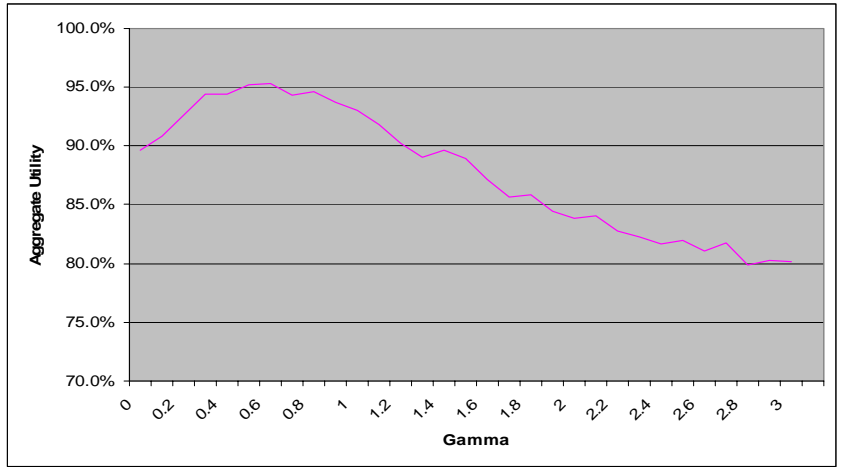

Fig. 3. Fully connected network with 12 nodes: aggregate utility.

Since simulation of one scenario (one set of parameters, including $\gamma$ ) takes approximately 11 hours, we looked at one sourcedestination pair, approximately accounting for the effect of the remainder of the network by fixed background traffic. Running one scenario for this simplified network model takes approximately one hour. To reduce simulation time further we investigated a simple two-node, two-link network shown on Figure 4.

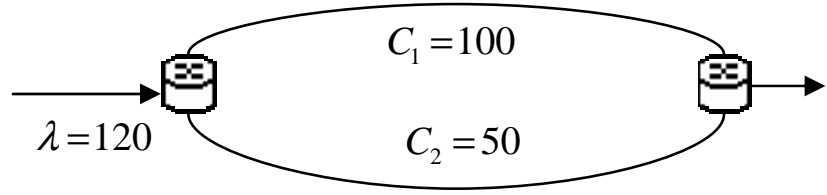

Fig. 4. Two-node, two-link network fragment.

This network qualitatively exhibits the same pattern of behavior shown on Figure 3 with $\gamma^{\text {opt }} \approx 0.7$.

Figure 5 shows evolution of the low capacity link cost for sufficiently high level of randomness in the route selection: 
$0 \leq \gamma<<\gamma^{\text {opt }}$. This evolution exhibits stable routing behavior without periodic pattern indicative of a route flapping instability. Random fluctuations in the link cost result from random flow arrivals/departures. Note that we cut off the link costs at certain level, arbitrarily scaled as link cost $d=1$.

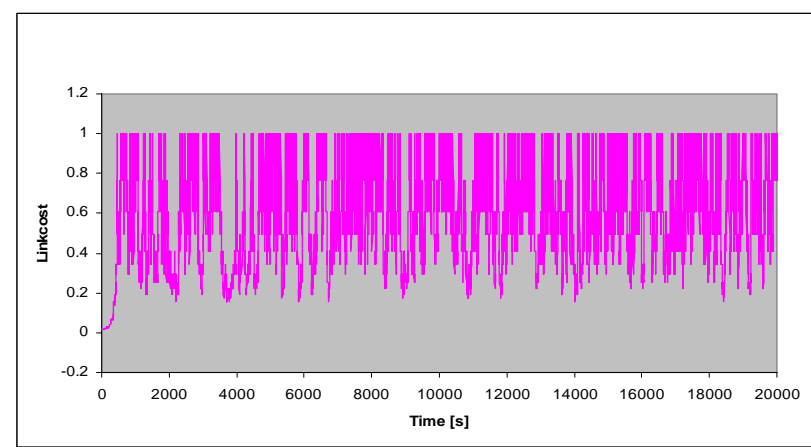

Fig. 5. Link costs: a case of stable routing $\left(0 \leq \gamma<<\gamma^{\text {opt }}\right)$

Figure 6 shows evolution of the low capacity link cost for sufficiently low level of randomness in the route selection: $\gamma>\gamma^{\text {opt }}$. This evolution demonstrates unstable (periodic) routing behavior combined with random fluctuations resulted from random flow arrivals/departures.

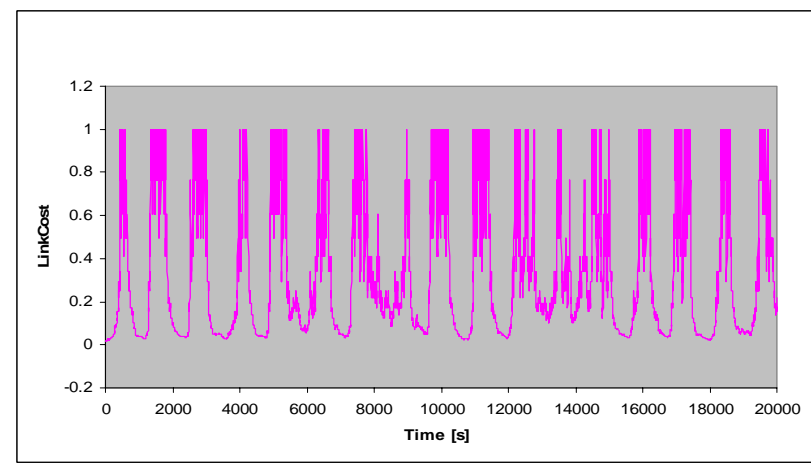

Fig. 6. Link costs: a case of unstable routing $\left(\gamma>>\gamma^{\text {opt }}\right)$

Figure 7 shows evolution of the low capacity link cost on the border between stability and instability regions: $\gamma \approx \gamma^{\text {opt }}$.

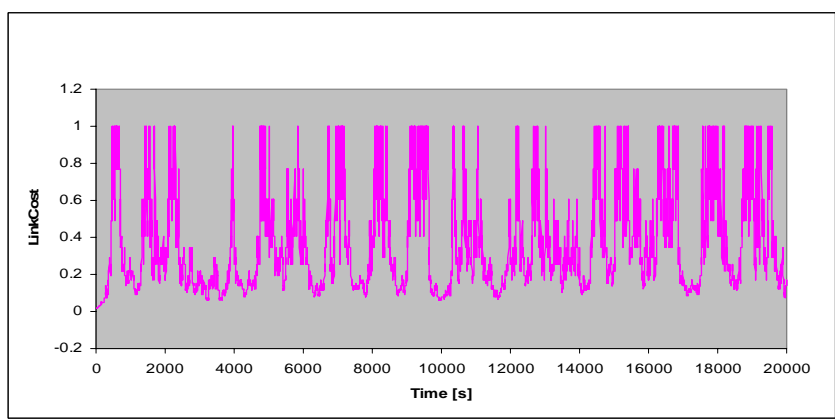

Fig. 7. Link costs: stability/instability border region $\left(\gamma \approx \gamma^{o p t}\right)$
Figure 7 demonstrates emergence of unstable (periodic) pattern in the routing behavior.

Simulation results presented on Figures 5-7 suggest that the optimal operating point lies within the network stability region in close proximity to the border of this region as assumed in off-line selection of the optimal operating point is depicted on Figure 2. We attempted to use this observation for adaptive optimization of the minimum cost routing. Based on the last 10 link cost update periods, the adaptive version of the minimum average cost routing algorithm approximately determines if the routing behavior is stable (non-periodic) or unstable (periodic). In the former case adaptive algorithm increases parameter $\gamma$ by 10 per cent. In the latter case adaptive algorithm reduces parameter $\gamma$ by 50 per cent. Figure 8 shows the corresponding random walk performed by the parameter $\gamma$.

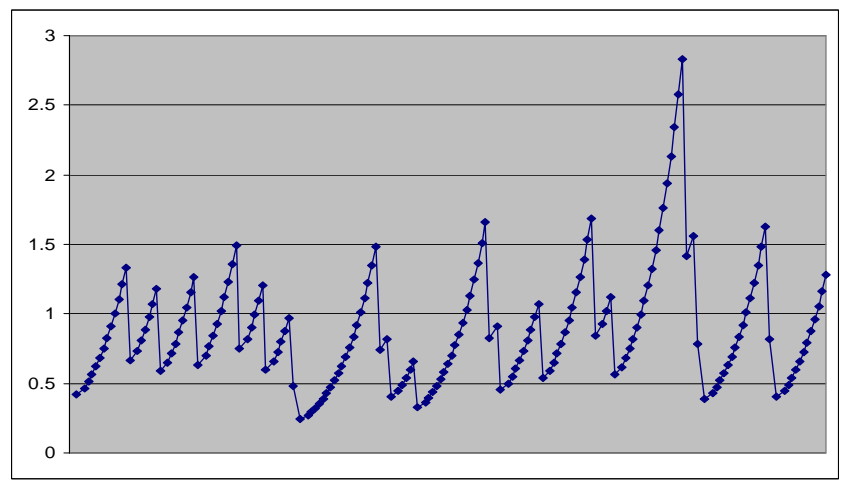

Fig. 8. Random walk by parameter $\gamma$.

Figure 9 demonstrates that the density of the probability distribution for this random walk is concentrated in the neighborhood of the optimal parameter $\gamma^{\text {opt }} \approx 0.7$. Simulation has also revealed that the performance loss for a case of the adaptive parameter $\gamma$ as compared to a case of preset optimal parameter $\gamma=\gamma^{\text {opt }}$ is only 2-3 per cent. This preliminary result may be viewed as an encouragement for future efforts in adaptive routing algorithms.

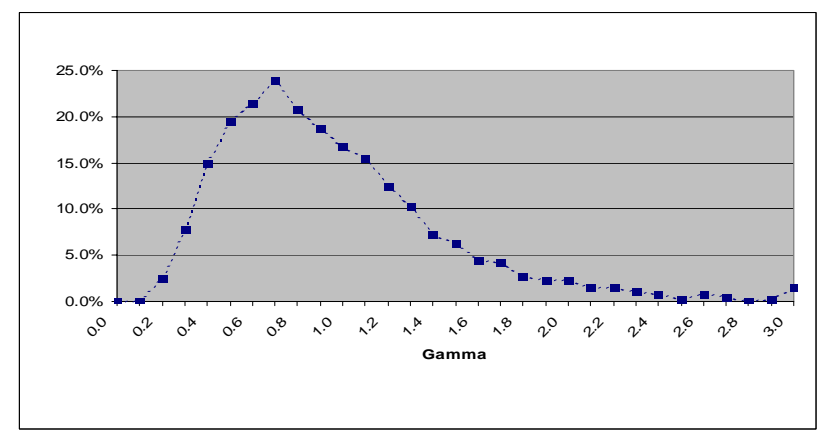

Fig. 9. Probability density of random walk by parameter $\gamma$. 


\section{CONCLUSION}

Based on interpretation of cross-layer optimization of TCP/IP network as a learning algorithm, this paper has reported initial simulation results indicating that (a) sufficient level of randomness in route selection improves the network performance by eliminating the route flapping instability, (b) the optimal level of randomness keeps the network within the stability region in close proximity to the border of this region, and (c) it may be possible to optimize the routing algorithm performance by adaptively the level of randomness in the route selection. Current research investigates shape of the stability region and the optimal selection of the operating point conjectured in Figure 2. This research is based on stability analysis of the discrete dynamic system (34)-(40). Future efforts should be concentrated on online determination of the set of "almost" minimum cost routes and estimation of the entropy of the route selection, based on the historic observations.

\section{ACKNOWLEDGMENTS}

The first author appreciates useful and spirit uplifting discussions with M. Carson and A. Nakassis.

\section{REFERENCES}

[1] A.G. Ganek and T.A. Corbi, "The dawning of the autonomic computing era,” IBM Systems J., Vol. 42, No 1, 2003, pp.518.

[2] D. Fudenberg and D.K. Levine, The theory of learning in games, The MIT Press, 1999.

[3] C. Huitema, Routing in the Internet, Prentice Hall, 2000.

[4] D. Bertsekas and R. Gallager, Data Networks, Prentice-Hall, New Jersey, 1992.

[5] J. Wang, L. Li, S.H. Low, and J.C. Doyle, "Cross-layer optimization in TCP/IP networks," IEEE/ACM Trans. on Networking, to appear, available at http://netlab.caltech.edu/.
[6] A. Elwalid, C. Jin, S. Low and J. Widjaja, "MATE: MPLS Adaptive Traffic Engineering," in Proceedings of Infocom'2001, Anchorage, Alaska, April 2001.

[7] D.P. Bertsekas, "Dynamic behavior of shortest path routing algorithm for communication networks," IEEE Trans. On Automatic Control, pp. 60-74, Feb. 1982.

[8] S.H. Low and P. Varaiya, "Dynamic behavior of a class of adaptive routing protocols (IGRP),” Proc. Of Infocom'93, pp. 610-616, March 1993.

[9] Z. Wang, Y. Wang, and L. Zhang, "Internet traffic engineering without full mesh overlaying," in Proceedings of Infocom'2001, Anchorage, Alaska, April 2001.

[10]A. Sridharan, R. Guerin, and Cristophe Diot, “Achieving Near Optimal Traffic Engineering Solution for Current OSPF/IS-IS Networks," in Proceedings of Infocom'2003.

[11] F.P. Kelly, A.K. Maulloo, and D.H.K. Tan, “The rate control for communication networks: shadow prices, proportional fairness and stability," Journal of the Operational Research Society, pp. 237-252, vol. 409, 1998.

[12] T.M. Cover and J.A. Thomas, Elements of Information Theory, Wiley, 1991.

[13] V. Marbukh, "Network provisioning as a game against nature," IEEE International Communications Conference (ICC 2003), Anchorage, AK, 2003.

[14] V. Marbukh, “On Shortest random walks under adversarial uncertainty", Fortieth Annual Allerton Conference on Communication, Control, and Computing, Monticello, Illinois, 2002.

[15] O. Borchert and R. Rouil, "The GMPLS lightwave agile switching simulator - $\quad$ an overview," http://www.antd.nist.gov/glass. 